\title{
Efficacy and safety of duloxetine for postoperative pain after total knee arthroplasty in centrally sensitized patients: study protocol for a randomized controlled trial
}

Shicheng Wang ${ }^{1}$, Wensheng Wang ${ }^{2}$, Long Shao ${ }^{1}$ and Jing Ling ${ }^{1 *}$

\begin{abstract}
Background: Postoperative residual knee pain after total knee arthroplasty (TKA) is a significant factor that contributes to patient dissatisfaction. Patients with preoperative central sensitization (CS) may be more susceptible to unexplained chronic pain after TKA, and duloxetine has been reported to be effective in post-TKA pain control in patients with CS. However, there remains limited evidence to support this off-label use in routine clinical practice. Hence, we designed this randomized, placebo-controlled, triple-blind clinical trial to evaluate the effects of preoperative screening and targeted duloxetine treatment of CS on postoperative residual pain compared with the care-as-usual control group.
\end{abstract}

Methods: This randomized controlled trial includes patients with knee osteoarthritis on a waiting list for primary unilateral TKA. Patients with preoperative CS will be randomly allocated to the perioperative duloxetine treatment group (duloxetine group) or the care-as-usual control group (placebo group). Patients in the duloxetine group will receive a half-dose of preemptive duloxetine (30 mg/day) for a week before surgery and a full-dose of duloxetine ( $60 \mathrm{mg} /$ day) for six weeks after surgery. The primary outcome is the intensity of residual pain at six months after TKA, including the visual analogue scale, 11-point numeric rating scale, the sensory dimension of the brief pain inventory, and the pain subscale of the Knee injury and Osteoarthritis Outcome Score. The secondary outcome measures will include the pain and function related outcomes. All of the patients will be followed up at one, three, and six months after surgery. All adverse events will be recorded and immediately reported to the primary investigator and ethics committee to decide if the patient needs to drop out from the trial.

Discussion: This clinical trial will convey the latest evidence of the efficacy and safety of the application of duloxetine in postoperative pain control in CS patients who are scheduled for TKA. The study results will be disseminated at national and international conferences and published in peer-reviewed journals.

Trial registration: Chinese Clinical Trial Registry (http://www.chictr.org.cn) registration number: ChiCTR2000031674. Registered 07 April 2020.

Keywords: Central sensitization, Duloxetine, Pain, Total knee arthroplasty

\footnotetext{
* Correspondence: lingjing82@126.com

'Department of Orthopaedic Surgery, Ningbo No.6 Hospital, 1059

Zhongshan East Road, Ningbo 315040, Zhejiang, China

Full list of author information is available at the end of the article
}

(c) The Author(s). 2021 Open Access This article is licensed under a Creative Commons Attribution 4.0 International License, which permits use, sharing, adaptation, distribution and reproduction in any medium or format, as long as you give appropriate credit to the original author(s) and the source, provide a link to the Creative Commons licence, and indicate if changes were made. The images or other third party material in this article are included in the article's Creative Commons licence, unless indicated otherwise in a credit line to the material. If material is not included in the article's Creative Commons licence and your intended use is not permitted by statutory regulation or exceeds the permitted use, you will need to obtain permission directly from the copyright holder. To view a copy of this licence, visit http://creativecommons.org/licenses/by/4.0/. The Creative Commons Public Domain Dedication waiver (http://creativecommons.org/publicdomain/zero/1.0/) applies to the data made available in this article, unless otherwise stated in a credit line to the data. 


\section{Background}

Total knee arthroplasty (TKA) is one of the most successful and effective surgical options performed in orthopedics, aiming to relieve pain and restore function for patients with end-stage knee osteoarthritis (OA) [1]. Over the past decades, significant advances have been made in TKA surgical techniques, implant design, patient selection, and perioperative care management, which led to the continuous improvement of clinical outcomes and expanded surgical indications [2-16]. Given the rapidly aging population and the growing prevalence of knee-related diseases such as OA and rheumatoid arthritis, the number of TKA procedures is projected to increase substantially worldwide [17-19]. However, the dissatisfaction rate following a TKA has persisted over the last decade, with approximately $10 \% \sim 20 \%$ of patients remain dissatisfied following primary TKA [20-23]. As patient satisfaction has been considered a key parameter in assessing success after TKA, a substantial proportion of patients failed to achieve the desired goal of surgery [22]. Therefore, patient dissatisfaction following TKA remains a challenging problem and should be a high priority.

Previous studies have investigated the causes of dissatisfaction after TKA, and the most common reasons were residual pain and limited function [22, 24-27]. A significant number of patients remain dissatisfied as the persistent chronic pain was not relieved, even though the TKA procedure has removed the pain source [28, 29]. Recent studies found that the pain in patients with OA varies from nociceptive to neuropathic pain-(NP) like symptoms, which may contribute to joint nociceptors' exposure to the changing biochemical environment during the process of OA [30]. It is thought that these changes might contribute to the activation and sensitization of nociceptors, lead to hyperexcitability of the central nervous system (central sensitization, CS) [29-32]. CS is defined as increased responsiveness of nociceptive neurons in the central nervous system, which is characterized by pain in the presence of a nonnoxious stimulus (allodynia) and pain hypersensitivity (hyperalgesia) [33]. Not all patients with knee OA are suffered from CS, but the subgroup of patients with CS is related to low pain thresholds, high level of preoperative pain, and severe pain in the early postoperative period after TKA [34-36]. Furthermore, preoperative CS has been recently recognized as a significant risk factor for persistent pain and dissatisfaction following TKA, which could persist two years after TKA, resulting in worse quality of life, functional disability, and dissatisfaction [28, 34, 37-40]. Considering postoperative residual pain is the primary cause of dissatisfaction following TKA and CS has been identified as a risk factor for persistent postoperative pain and dissatisfaction, preoperative screening and treatment of CS could be an effective way to decrease the level of residual pain after TKA and improve patient satisfaction.

The treatment options for CS focus on those strategies that specifically target the pathophysiological mechanisms known to be involved in CS, which aim to desensitize the central nervous system. The treatment options mainly include pharmacological options (acetaminophen, serotonin-reuptake inhibitor drugs, selective and balanced serotonin and norepinephrine-reuptake inhibitor (SNRI) drugs, serotonin precursor tryptophan, opioids, N-methyl$\mathrm{d}$-aspartate (NMDA)-receptor antagonists, calciumchannel alpha(2)delta (a2 $\delta$ ) ligands), rehabilitation (e.g., transcutaneous electric nerve stimulation (TENS), manual therapy) and neurotechnology options (e.g., transcranial magnetic stimulation) $[41,42]$. Ho et al. performed a randomized controlled trial (RCT) to evaluate the efficacy of duloxetine in reducing morphine requirements in patients after TKA and found that perioperative administration of duloxetine could reduce postoperative morphine requirements during the first $48 \mathrm{~h}$ after TKA, without significant differences in pain scores or adverse effects [43]. YaDeau et al. conducted a triple-blinded RCT to evaluate the efficacy of duloxetine on subacute pain after TKA, in which patients received $60 \mathrm{mg} /$ day from the day of surgery for 14 days [44]. They found that duloxetine did not reduce pain at rest, with ambulation or flexion, but duloxetine could reduce opioid consumption and nausea in the first 14 days after surgery [44]. Compared with the normal controls, patients with CS are associated with more severe postoperative pain, greater opioid consumption, and a higher risk of persistent pain, so the analgesic efficacy of peri-TKA duloxetine could be more effective in patients with CS. Koh et al. conducted an RCT in patients with preoperative CS to investigate whether duloxetine could reduce postoperative pain and improve recovery quality after TKA [32]. This study suggested that in patients with preoperatively identified CS, duloxetine reduced postoperative pain and improved quality of recovery, without increasing the risk of adverse medication effects following TKA [32].

Based on previous research, for patients who are identified as having CS before TKA, supplementing perioperative duloxetine to the multimodal analgesic protocol may help to reduce postoperative pain and opioid consumption, improve the quality of recovery in terms of both emotional and physical functioning, without increasing the risk of adverse events [32, 43, 44]. However, at this time, there is insufficient clinical data to recommend routine use of duloxetine in patients with CS to ameliorate the unexplained postoperative pain after TKA. In order to tackle this knowledge gap, we designed this prospective randomized study to evaluate the efficacy and safety of preoperative screening and targeted 
duloxetine treatment of CS on residual pain compared with the care-as-usual control group. We hypothesized that perioperative duloxetine would reduce postoperative pain and analgesic consumption, and enhance postoperative functional recovery after TKA, without increasing the risk of adverse events in patients who are identified as having CS before TKA. We present the following article in accordance with the Standard Protocol Items: Recommendations for Interventional Trials (SPIRIT) reporting checklist [45].

\section{Methods}

\section{Study design}

This prospective, randomized clinical trial was designed as a single-center parallel-group study with balanced randomization and enrolled patients who are scheduled for TKA. This protocol is reported following the SPIRIT statement [45]. This study was approved by our institutional review board and was registered at the Chinese Clinical Trial Registry (http://www.chictr.org.cn) (ChiCTR2000031674). Ethical approval for this retrospective cohort study was obtained from the institutional ethical committee (K2020005). The trial flow chart is shown in Fig. 1.

\section{Participants}

All potentially eligible participants will be selected from the consecutively recruited patients who agreed to participate. According to the inclusion and exclusion criteria, one of the authors will screen the potentially qualified participants.

\section{Inclusion criteria}

Participants who fulfill the following criteria will be included: (I) Patients scheduled to undergo primary unilateral TKA for primary OA of the knee; (II) aged 50 years or older (CS are more prevalent in patients with a long history of knee OA); (III) had education above primary school; (VI) American Society of Anesthesiologists (ASA) physical status grade I $\sim$ III; (V) participants who voluntarily participate in the study and sign a written informed consent form.

\section{Exclusion criteria}

Candidates who meet any of the following criteria will be excluded from participation:

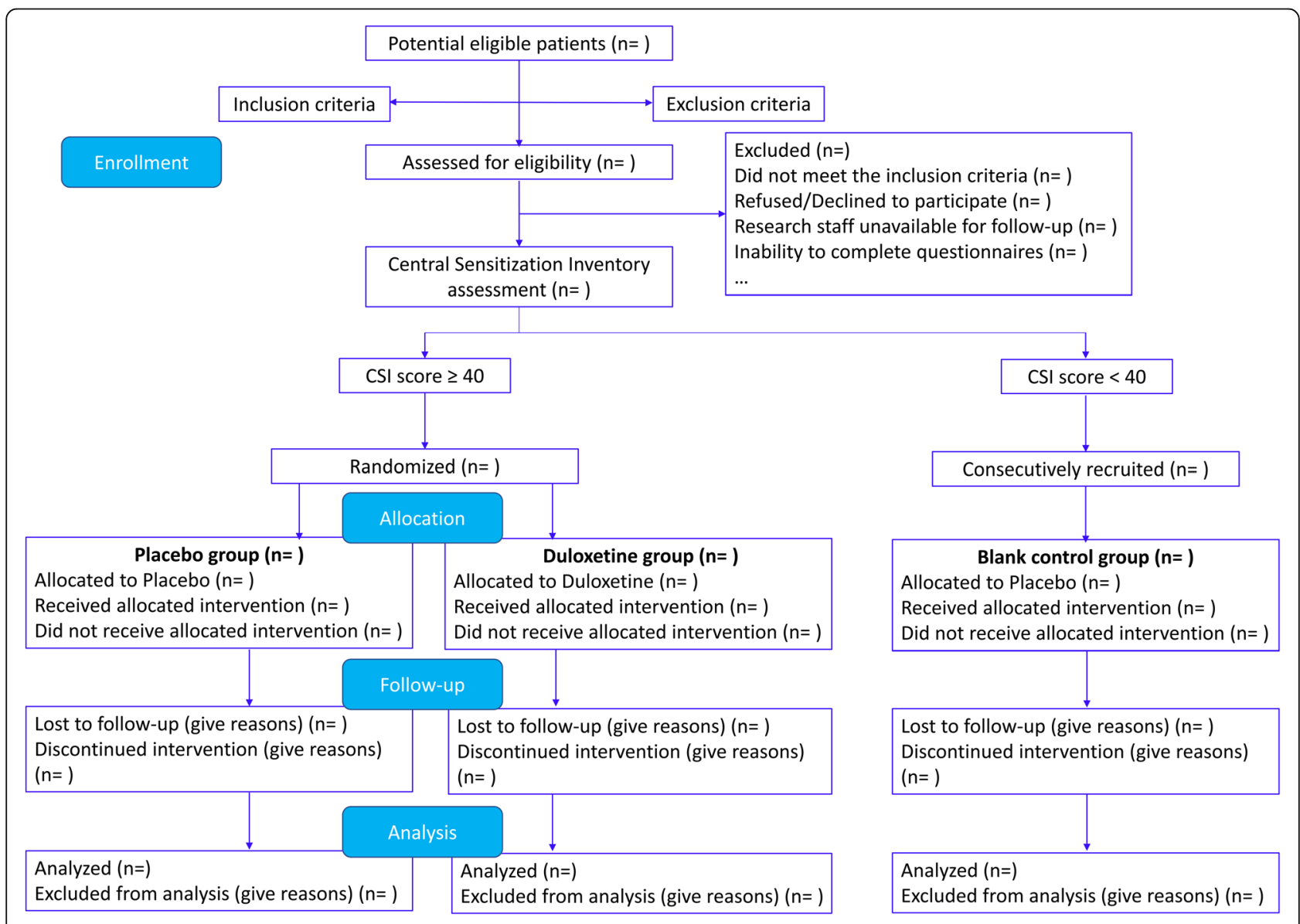

Fig. 1 CONSORT 2010 flow diagram of the study sample 


\section{General exclusion criteria}

(I) Received surgical knee joint procedures in the past year; (II) intra-articular knee injection or knee arthroscopy in the past three months; (III) received other surgical procedure (e.g., hip joint procedure, major thoracic or abdominal operations that may influence the assessment of CS) during the past year; (VI) planned or intended contralateral TKA procedure or any other surgical procedure within the study duration; (V) cognitive or neurological disorders that could strongly affect the questionnaire surveys (e.g., Alzheimer's disease, dementia); (VI) inability to complete research questionnaires; (VII) with severe preoperative comorbidities that are more likely to be hospitalized during the course of the study or the illness compromises study participation significantly; (VIII) pre-existing pain (except the knee joint pain) and received relevant analgesic treatment; (IX) patients who refused to participate in the study due to stress events; $(\mathrm{X})$ rejection of randomization.

\section{Duloxetine-related exclusion criteria}

(I) Previous exposure to duloxetine, non-selective monoamine oxidase inhibitors, tricyclic antidepressants, selective serotonin reuptake inhibitors or SNRIs; (II) allergy to the duloxetine; (III) simultaneously usage of strong cytochrome P450 1A2 (CYP1A2) inhibitors; (IV) impaired liver function, liver cirrhosis, or liver transplantation; (V) severe renal impairment (estimated creatinine clearance less than $30 \mathrm{ml} / \mathrm{min}$ ), requiring renal dialysis, or renal transplantation; (VI) history of cardiac arrhythmias, cardiac failure, myocardial infarction; (VII) personality disorder, psychiatric disorders or any major depressive disorder (based on Hospital Anxiety and Depression Scale (HADS), the cut-off point score was $\geq 11$ ) [46]; (VIII) chronic gabapentin or pregabalin use (regular use for longer than 3 months), and chronic opioid use (regular use for longer than 3 months); (IX) a history of alcohol or other substance abuse or dependence prior to enrolment; (X) any other possible conditions that are considered inappropriate to participant in this clinical trial.

\section{Randomization and allocation}

After applying the selection criteria, the potentially eligible patients will be screened with the Central Sensitization Inventory (CSI) preoperatively, with a CSI score $\geq 40$ indicating CS and a CSI score of $<40$ indicating normal [4648]. Patients with preoperative CS will be randomly allocated (1:1) to the duloxetine group or the placebo group by a computer-generated randomization list. After randomization, there will be a baseline assessment, such as patient demographics and baseline values for outcome measures. In addition, a group of patients who are assessed as normal will receive the placebo (as the blank control group). The hospital pharmacy will prepare indistinguishable capsules containing either duloxetine or placebo for the study. Randomization and allocation will be revealed only after the required number of subjects has been recruited and the data analysis has been completed.

\section{Blinding}

This trial was designed as a pragmatic, randomized, controlled, triple-blinded trial with three parallel arms. All patients, surgeons, clinical investigators who are responsible for data collection, and statisticians will be kept unaware of group assignments until the final data analyses were completed. Although patients will be kept blind to their prescription, the computer-generated randomization table will be provided to the hospital pharmacy. Therefore, our hospital pharmacy will not be blinded to the randomization and will prepare all medications with a single packet of medicine as scheduled. Considering the success of blinding is a fundamental issue in clinical trials, blinding will be further verified with the Bang's Blinding Index [49]. After the intervention, patients will answer a separate question ("Guess Duloxetine", "Guess Placebo", "Don't Know") in the blind assessor questionnaire, which attempts to measure the effectiveness of blinding.

\section{Perioperative care}

All TKAs will be performed by the same orthopedic surgeon using the same approach. The routine perioperative analgesic strategy, including preemptive analgesia (celecoxib), intraoperative periarticular cocktail injection, postoperative patient-controlled analgesia (PCA) and celecoxib, and discharge medication (celecoxib). In case of patients suffering unbearable postoperative pain, morphine or other opioids will also be given, the opioid consumption would also be recorded and compared. In the duloxetine group, patients will receive a half-dose of preemptive duloxetine (30 $\mathrm{mg} /$ day) for a week before surgery, and a full-dose of duloxetine $(60 \mathrm{mg} /$ day $)$ for six weeks after surgery. The maintenance dose $(60 \mathrm{mg} /$ day $)$ of duloxetine was chosen based on previous publications $[32,43,44,50]$. While patients in the placebo group and blank control group will receive placebo for the same period. All enrolled patients will receive the same standard preoperative publicizing and education, intraoperative type of anesthesia, postoperative care, and rehabilitation.

\section{Follow-up period}

During hospitalization, the basic metabolic panel will be obtained once or more to monitor serum sodium level, as hyponatremia is a duloxetine-induced complication that may occur early on after duloxetine initiation. In our routine clinical practice, patients are advised to 
return to the outpatient clinic for follow-up evaluation at one, three, and six months after surgery and subsequently every one year after surgery. Thus, in this clinical trial, all of the patients will be followed up at one, three, and six months after surgery. If patients are unwilling or unable to return to the study center for evaluation, they will be interviewed via telephone. Both the hospital and outpatient follow-up evaluation will be conducted by an investigator who is blinded to the experimental groups.

\section{Criteria for withdrawal}

Participants will be informed that they are under no obligation to participate and have the right to withdraw consent for participation in this clinical trial at any point without prejudice, and their routine clinical care and follow-up will not be affected by declining to participate or withdrawing from the trial. Meanwhile, the investigator or regulatory authority can discontinue a patient's participation in the clinical trial at any time if medically or otherwise necessary. Of note, it is not recommended to discontinue duloxetine abruptly, especially when taking the maintenance dose of $60 \mathrm{mg} /$ day. Therefore, the participant who wishes to discontinue or withdraw must contact the investigator to obtain discontinuation advice.

\section{Outcome measurement}

The following outcome parameters and patient characteristics will be retrieved from electronic hospital information systems or medical records, physical examination, and patient questionnaires.

\section{Primary outcome measures}

The primary outcome is the intensity of residual pain at six months after TKA. The perception of residual pain will be measured with the visual analogue scale (VAS), 11-point numeric rating scale (NRS), the sensory dimension of the Brief Pain Inventory (BPI), and the pain subscale of the Knee injury and Osteoarthritis Outcome Score (KOOS) at six months after surgery [51-54]. These questionnaires have been proven to be valid and reliable, and the results of these questionnaires will help to ascertain the robustness of our results. The key postoperative time point of six months was chosen as this is recognized as the first possible time point to evaluate the 'success' of TKA in clinical practice.

\section{Secondary outcome measures}

The secondary outcome measures will include the following. The pain-related outcomes mainly included the cumulative PCA consumption during the first $48 \mathrm{~h}$ after TKA and the amounts of rescue analgesic at $48 \mathrm{~h}$ after surgery. We will also evaluate the pain severity using the VAS score, NRS score, BPI score, and KOOS score. The function-related outcomes included knee range of motion (ROM), Knee Society Score (KSS) score, Western Ontario and McMaster Universities Arthritis Index (WOMAC) score, and physical activity [55]. These outcomes will be assessed by means of several questionnaires at multiple follow-up time points. Furthermore, we will also collect other parameters, including jointassociated problems, health-related quality of life, CSI score, depressive and anxiety symptoms, perceived improvement and arthroplasty-related expectations, and patient satisfaction (ordinal scale) [56].

\section{Patient characteristics}

The following baseline descriptive data will be obtained: age, gender, height $(\mathrm{cm})$ and weight $(\mathrm{kg})$, body mass index (BMI), marital status, family status, education level, employment status, duration of OA pain symptoms, Kellgren-Lawrence (K-L) grade, OA pain-related medication consumption, ASA classification, smoking and alcohol consumption, disease history, comorbidities, history of drug use, and past health problems.

\section{TKA-related characteristics}

The surgical procedure-related data include the type of anesthesia, surgical approach, type of implant, operation time, intraoperative and postoperative blood loss, and arthroplasty-related complications.

\section{Adverse events}

All adverse events reported spontaneously by the patients or observed by the investigators or staff will be recorded. If any adverse event occurs, the doctor will provide the corresponding treatment to the patient. Meantime, the adverse events will be immediately reported to the primary investigator and ethics committee to decide if the patient needs to withdraw from the trial. Based on previous literature, the most common adverse events were decreased appetite, nausea and vomiting, fatigue, insomnia, constipation, and dry mouth, which could be well managed with i.v. ondansetron [32, 43, 44].

\section{Data collection and management}

The demographic and baseline characteristic data will be collected by screeners when the patients are recruited and enrolled in this trial (Table 1). During the hospitalization and follow-up periods, the clinical outcomes, questionnaires, incidence of complications, and adverse events will be collected by an independent trained investigator and will be monitored by an independent Data Monitoring Committee. No additional participants will be included during the re-evaluation period. Personal data will be handled confidentially. Every participant will receive a unique code, and data of each patient will be collected under this unique code. A unique patient identification list will be used to link the data to the patient, and the key to the 
Table 1 Demographic and preoperative clinical characteristics of the patients

\begin{tabular}{|c|c|c|c|}
\hline Demographics & $\begin{array}{l}\text { Placebo } \\
\text { group }\end{array}$ & $\begin{array}{l}\text { Duloxetine } \\
\text { group }\end{array}$ & $\begin{array}{l}\text { Blank control } \\
\text { group }\end{array}$ \\
\hline \multicolumn{4}{|l|}{ Age (yr) } \\
\hline \multicolumn{4}{|l|}{ Gender, n (\%) } \\
\hline \multicolumn{4}{|l|}{ Male } \\
\hline \multicolumn{4}{|l|}{ Female } \\
\hline \multicolumn{4}{|l|}{$\mathrm{BMI}\left(\mathrm{kg} / \mathrm{m}^{2}\right)$} \\
\hline \multicolumn{4}{|l|}{ Marital status } \\
\hline \multicolumn{4}{|l|}{ Family status } \\
\hline \multicolumn{4}{|l|}{ Education level, n (\%) } \\
\hline \multicolumn{4}{|l|}{ Primary school } \\
\hline \multicolumn{4}{|l|}{ High school } \\
\hline \multicolumn{4}{|l|}{ Some college } \\
\hline \multicolumn{4}{|l|}{$\begin{array}{l}\text { Technical degree/ } \\
\text { associate's degree }\end{array}$} \\
\hline \multicolumn{4}{|l|}{ Bachelor's degree } \\
\hline \multicolumn{4}{|l|}{$\begin{array}{l}\text { Advanced/professional } \\
\text { degree (MA, PhD, etc.) }\end{array}$} \\
\hline \multicolumn{4}{|l|}{ Employment status } \\
\hline \multicolumn{4}{|l|}{ ASA status } \\
\hline \multicolumn{4}{|l|}{ । } \\
\hline \multicolumn{4}{|l|}{$\|$} \\
\hline \multicolumn{4}{|l|}{ III } \\
\hline \multicolumn{4}{|l|}{ Preoperative parameters } \\
\hline \multicolumn{4}{|l|}{$\begin{array}{l}\text { Duration of OA pain } \\
\text { symptoms }\end{array}$} \\
\hline \multicolumn{4}{|l|}{ Kellgren-Lawrence grade } \\
\hline \multicolumn{4}{|l|}{$\begin{array}{l}\text { OA pain-related medication } \\
\text { consumption }\end{array}$} \\
\hline \multicolumn{4}{|l|}{ Smoking consumption } \\
\hline \multicolumn{4}{|l|}{ Alcohol consumption } \\
\hline \multicolumn{4}{|l|}{ History of drug use } \\
\hline \multicolumn{4}{|l|}{ CSI score } \\
\hline \multicolumn{4}{|l|}{ VAS score } \\
\hline \multicolumn{4}{|l|}{ NRS score } \\
\hline \multicolumn{4}{|l|}{ BPI-pain severity score } \\
\hline KOOS score & & & \\
\hline
\end{tabular}

ASA American Society of Anesthesiologists, BMI Body Mass Index, BPI Brief Pain Inventory, CSI Central Sensitization Inventory, KOOS Knee injury and Osteoarthritis Outcome Score, MA Master of Arts, NRS numeric rating scale, $O A$ Osteoarthritis, VAS visual analogue scale

code will be safeguarded by the principal investigator. All source documents will be entered into the trial database (OpenClinica clinical trials software).

\section{Sample size}

Sample size calculation was performed with the VAS score as the primary outcome measure. Based on the previous study, the smallest change score for the VAS score to be considered clinically relevant is 2 points (on a $0-10$ scale) between the duloxetine group and the placebo group [32]. The power calculation is performed based on the VAS score difference using a two-sided hypothesis test at an alpha level of 0.05 and a power of $80 \%$, and a total of 38 participants is needed in each group [57-59]. Taking into account the possibility of $20 \%$ violators or dropouts, we will include 50 patients in each group.

\section{Statistical analysis}

Analyses will be performed using the IBM SPSS software (version 21.0, IBM Corp., New York, NY, USA). All outcomes measures will be assessed using both the ITT (intention-to-treat, all randomly assigned patients) and PP (per-protocol, patients who completed the trial without any protocol deviations) data sets. The missing value will be imputed by the last-observation-carried-forward (LOCF) method. For continuous variables and descriptive values, means \pm standard deviations (SDs) will be reported. For the enumerative variables, frequency and corresponding percentage will be calculated. For the variables with a normal distribution, statistical comparisons between the groups will be made by using a $t$-test. For variables with a non-normal distribution or ordinal level, the statistical comparison will be made using the MannWhitney U test. Outcomes with a discrete distribution will be expressed as percentages and analyzed by the chi-squared test or Fisher's exact test as appropriate. A $P$ value $<0.05$ is considered statistically significant.

\section{Discussion}

Residual pain is the most common reason for patient dissatisfaction following TKA. Recently, several studies suggested that CS is associated with chronic postoperative pain and decreased satisfaction after TKA. Duloxetine has been used in an off-label way to treat centrally mediated chronic pain in patients with preoperative CS, but the efficacy of pain control after TKA remains unclear. Currently, the available evidence is extremely limited; thus, we designed this clinical trial to examine the efficacy and safety of duloxetine for postoperative pain after TKA in CS patients. The present study was designed based on previous literature, thus, our trial has some advances and strengthens when compared with previous similar RCTs [32]. First of all, except the duloxetine group and placebo group, we set an extra blank control group, which consists of normal patients without medication. Parents in the blank control group will provide the standard baseline for the trial, which will help to evaluate the magnitude of improvement. Second, several parameters will be included to measure the intensity of residual pain, including the VAS score, NRS score, 
BPI score, and KOOS score. The consistency of these parameters will help to ascertain the robustness of the results. Third, based on previous RCTs, we will collect all of the efficacy and safety related parameters, which will contribute to a more comprehensive evaluation of duloxetine.

We will ensure that this study is a genuinely randomized, controlled, triple-blinded trial through the full implementation of randomization, blindness, and concealment. We will conduct and report this clinical trial in strict accordance with the Consolidated Standards of Reporting Trials (CONSORT) [60]. It is important to acknowledge that the results of this trial will provide scientific and rigorous clinical evidence for the application of perioperative duloxetine in CS patients being scheduled for TKA.

\section{Abbreviations \\ ASA: American Society of Anesthesiologists; BMI: Body Mass Index; BPI: Brief Pain Inventory; CONSORT: Consolidated Standards of Reporting Trials; CS: Central Sensitization; CSI: Central Sensitization Inventory; HADS: Hospital Anxiety and Depression Scale; ITT: Intention-To-Treat; K-L: Kellgren-Lawrence; KOOS: Knee injury and Osteoarthritis Outcome Score; KSS: Knee Society Score; LOCF: Last-Observation-Carried-Forward; MA: Master of Arts; NRS: Numeric Rating Scale; NMDA: N-methyl-d-aspartate; OA: Osteoarthritis; PCA: Patient-Controlled Analgesia.; PP: Per-Protocol.; RCT: Randomized Controlled Trial.; ROM: Range Of Motion; SD: Standard Deviation; \\ SNRI: Selective and balanced serotonin and Norepinephrine-Reuptake Inhibi- tor; SPIRIT: Standard Protocol Items Recommendations for Interventional Trials; TENS: Transcutaneous Electric Nerve Stimulation; TKA: Total Knee Arthroplasty; VAS: Visual Analogue Scale; WOMAC: Western Ontario and McMaster Universities Arthritis Index}

\section{Acknowledgements}

The authors thank all patients who participated in this study for their contribution.

\section{Study status}

This project is delayed because of the Covid-19 pandemic. Recruitment is scheduled to begin on $30^{\text {th }}$ March 2021, and the study will continue until $28^{\text {th }}$ Feb 2022

\section{Related articles}

No publications containing the results of this study that have already been published or submitted to any journal.

\section{Authors' contributions}

SW and JL conceived the study while SW, LS and JL designed the study. The study protocol was drafted by SW, WW, LS, and revised by JL. All authors approved the final manuscript of this study protocol.

\section{Funding}

This work was supported by the Ningbo Medical Science and Technology Project (grant numbers 2019Y53), awarded to investigator Wensheng Wang. The funding body has no influence over the design, conduct, or reporting of the trial. Open Access funding provided by Ningbo No. 6 Hospital. The foundation conducted an external review of the study protocol before sponsored this project. Academic Board reviewed and discussed the grant application prior to voting on the final recipient to receive funding support After obtaining the research grant, the study proposal and protocol were further reviewed in Ningbo No. 6 Hospital institutional review board (IRB). The Board reviewed and discussed the scientific validity and ethical concerns of the study, and give final approval of the study protocol.

Availability of data and materials Not applicable.

\section{Declarations}

\section{Ethics approval and consent to participate}

The authors are accountable for all aspects of the work in ensuring that questions related to the accuracy or integrity of any part of the work are appropriately investigated and resolved. The study was conducted in accordance with the Declaration of Helsinki (as revised in 2013). The study protocol was approved by the Ethics Committee of Ningbo No. 6 Hospital (K2020005). Informed consent will be obtained from each patient. The findings of this study will be disseminated widely at national and international conferences, and will be published in peer-reviewed, scientific journals.

\section{Consent for publication}

All patients who voluntarily participate in the study will sign a written informed consent. Volunteers are aware of this publication and have clarified the importance of the study and publication of the manuscript.

\section{Competing interests}

All authors have completed the ICMJE uniform disclosure form. The authors declare that they have no competing interests.

\section{Author details}

${ }^{1}$ Department of Orthopaedic Surgery, Ningbo No.6 Hospital, 1059 Zhongshan East Road, Ningbo 315040," Zhejiang, China. ${ }^{2}$ Department of Neurology, Ningbo No.6 Hospital, 1059 Zhongshan East Road, Ningbo 315040, Zhejiang, China.

Received: 26 January 2021 Accepted: 11 March 2021

Published online: 30 March 2021

\section{References}

1. Harris WH, Sledge CB. Total hip and total knee replacement. N Engl J Med. 1990;323(11):725-31

2. McAllister CM, Stepanian JD. The impact of minimally invasive surgica techniques on early range of motion after primary total knee arthroplasty. J Arthroplast. 2008:23(1):10-8.

3. Rivière C, Iranpour F, Auvinet E, Howell S, Vendittoli P-A, Cobb J, Parratte S. Alignment options for total knee arthroplasty: a systematic review. Orthop Traumatol. 2017;103(7):1047-56.

4. Schiraldi M, Bonzanini G, Chirillo D, de Tullio V. Mechanical and kinematic alignment in total knee arthroplasty. Ann Transl Med. 2016;4(7):130-4.

5. Kurita M, Tomita T, Yamazaki T, Fujii M, Futai K, Shimizu N, Yoshikawa H, Sugamoto K. In vivo kinematics of high-flex mobile-bearing total knee arthroplasty, with a new post-cam design, in deep knee bending motion. Int Orthop. 2012:36(12):2465-71.

6. Sassoon A, Nam D, Nunley R, Barrack R. Systematic review of patient-specific instrumentation in total knee arthroplasty: new but not improved. Clin Orthop Related Res. 2015;473(1):151-8.

7. Wu X-D, Xiang B-Y, Schotanus MG, Liu Z-H, Chen Y, Huang W. CT-versus MRI-based patient-specific instrumentation for total knee arthroplasty: a systematic review and meta-analysis. Surgeon. 2017;15(6):336-48.

8. Scuderi GR, Tenholder M, Capeci C. Surgical Approaches in Mini-incision Total Knee Arthroplasty. Clin Orthop Related Res (1976-2007) 2004, 428 : $61-67$

9. Shao L, Wu X-D, Wang T, Liu X-K, Xu W, Huang W, Zeng Z-M. Approximating the maximum tibial coverage in total knee arthroplasty does not necessarily result in implant malrotation. Sci Rep. 2020;10(1):1-9.

10. Shao L, Wang T, Liao J, Xu W, Liang X, Huang W. Effect of tibial component alignment and posterior slope on tibial coverage in a Chinese population: A three-dimensional anthropometric study. J Knee Surg. 2020;33(01):053-61.

11. Cross WW III, Saleh KJ, Wilt TJ, Kane RL. Agreement about indications for total knee arthroplasty. Clin Orthop Relat Res. 2006;446:34-9.

12. Wu X-D, Tian M, He Y, Chen Y, Tao Y-Z, Shao L, Luo C, Xiao P-C, Zhu Z-L, Liu $J$-C. Efficacy of a three-day prolonged-course of multiple-dose versus a single-dose of tranexamic acid in total hip and knee arthroplasty. An Transl Med 2020, 8(6).

13. Auyong DB, Allen CJ, Pahang JA, Clabeaux JJ, MacDonald KM, Hanson NA Reduced length of hospitalization in primary total knee arthroplasty patients using an updated enhanced recovery after orthopedic surgery (ERAS) pathway. J Arthroplast. 2015;30(10):1705-9. 
14. Ibrahim MS, Khan MA, Nizam I, Haddad FS. Peri-operative interventions producing better functional outcomes and enhanced recovery following total hip and knee arthroplasty: an evidence-based review. BMC Med. 2013; 11(1):1-9.

15. Jauregui JJ, Cherian JJ, Pierce TP, Beaver WB, Issa K, Mont MA. Long-term survivorship and clinical outcomes following total knee arthroplasty. Arthroplast. 2015;30(12):2164-6.

16. Losina E, Paltiel AD, Weinstein AM, Yelin E, Hunter DJ, Chen SP, Klara K, Suter LG, Solomon DH, Burbine SA. Lifetime medical costs of knee osteoarthritis management in the United States: impact of extending indications for total knee arthroplasty. Arthritis Care Res. 2015;67(2):203-15.

17. Inacio M, Paxton E, Graves S, Namba R, Nemes S. Projected increase in total knee arthroplasty in the United States-an alternative projection model. Osteoarthritis cartilage. 2017;25(11):1797-803.

18. Kim TW, Kang S-B, Chang CB, Moon S-Y, Lee Y-K, Koo K-H. Current Trends and Projected Burden of Primary and Revision Total Knee Arthroplasty in Korea Between 2010 and 2030. The Journal of Arthroplasty. 2020;36(1):93-101.

19. Klug A, Gramlich $Y$, Rudert M, Drees P, Hoffmann R, Weißenberger M, Kutzner KP. The projected volume of primary and revision total knee arthroplasty will place an immense burden on future health care systems over the next 30 years. Knee Surg Sports Traumatol Arthroscopy 2020:1-12.

20. Robertsson O, Dunbar M, Pehrsson T, Knutson K, Lidgren L. Patient satisfaction after knee arthroplasty: a report on 27,372 knees operated on between 1981 and 1995 in Sweden. Acta Orthop Scand. 2000;71(3):262-7.

21. Bourne RB, Chesworth BM, Davis AM, Mahomed NN, Charron KD. Patient satisfaction after total knee arthroplasty: who is satisfied and who is not? Clin Orthop Related Res. 2010;468(1):57-63.

22. Gunaratne R, Pratt DN, Banda J, Fick DP, Khan RJ, Robertson BW. Patient dissatisfaction following total knee arthroplasty: a systematic review of the literature. J Arthroplast. 2017;32(12):3854-60.

23. Nakano N, Shoman H, Olavarria F, Matsumoto T, Kuroda R, Khanduja V. Why are patients dissatisfied following a total knee replacement? A systematic review. Int Orthop. 2020;44(10):1971-2007.

24. Halawi MJ, Jongbloed W, Baron S, Savoy L, Williams VJ, Cote MP. Patient dissatisfaction after primary total joint arthroplasty: the patient perspective. J Arthroplast. 2019;34(6):1093-6.

25. Choi Y-J, Ra HJ. Patient satisfaction after total knee arthroplasty. Knee Surg Related Res. 2016;28(1):1.

26. Bonnin MP, Basiglini L, Archbold HP. What are the factors of residual pain after uncomplicated TKA? Knee Surg Sports Traumatol Arthrosc. 2011;19(9): 1411-7.

27. Park CN, White PB, Meftah M, Ranawat AS, Ranawat CS. Diagnostic algorithm for residual pain after total knee arthroplasty. Orthopedics. 2016 39(2):e246-52

28. Wylde V, Hewlett $\mathrm{S}$, Learmonth ID, Dieppe P. Persistent pain after joint replacement: prevalence, sensory qualities, and postoperative determinants. PAIN $^{\circledast}$. 2011;152(3):566-72.

29. Kurien T, Arendt-Nielsen L, Petersen KK, Graven-Nielsen T, Scammell BE. Preoperative neuropathic pain-like symptoms and central pain mechanisms in knee osteoarthritis predicts poor outcome 6 months after total knee replacement surgery. J Pain. 2018;19(11):1329-41.

30. Malfait A-M, Schnitzer TJ. Towards a mechanism-based approach to pain management in osteoarthritis. Nat Rev Rheumatol. 2013;9(11):654.

31. Dimitroulas T, Duarte RV, Behura A, Kitas GD, Raphael JH. Neuropathic pain in osteoarthritis: a review of pathophysiological mechanisms and implications for treatment. In: Seminars in arthritis and rheumatism: 2014. Elsevier: 145-154.

32. Koh IJ, Kim MS, Sohn S, Song KY, Choi NY, In Y. Duloxetine reduces pain and improves quality of recovery following total knee arthroplasty in centrally sensitized patients: a prospective, randomized controlled study. JBJS. 2019;101(1):64-73.

33. Woolf CJ. Central sensitization: implications for the diagnosis and treatment of pain. Pain. 2011;152(3):2-15

34. Lundblad H, Kreicbergs A, Jansson K-å. Prediction of persistent pain after total knee replacement for osteoarthritis. J Bone Joint Surg Br. 2008;90(2): $166-71$

35. Kim SH, Yoon KB, Yoon DM, Yoo JH, Ahn KR. Influence of centrally mediated symptoms on postoperative pain in osteoarthritis patients undergoing total knee arthroplasty: a prospective observational evaluation. Pain Practice. 2015;15(6):E46-53.
36. Howells N, Murray J, Wylde V, Dieppe P, Blom A. Persistent pain after knee replacement: do factors associated with pain vary with degree of patient dissatisfaction? Osteoarthritis Cartilage. 2016;24(12):2061-8.

37. Wylde V, Palmer S, Learmonth I, Dieppe P. The association between preoperative pain sensitisation and chronic pain after knee replacement: an exploratory study. Osteoarthritis Cartilage. 2013;21(9):1253-6.

38. Kim MS, Koh IJ, Sohn S, Kang BM, Kwak DH, In Y. Central sensitization is a risk factor for persistent postoperative pain and dissatisfaction in patients undergoing revision total knee arthroplasty. J Arthroplast. 2019;34(8):1740-8.

39. Sigmund KJ, Hoeger Bement MK, Earl-Boehm JE. Exploring the pain in patellofemoral pain: A systematic review and meta-analysis examining signs of central sensitization. J Athletic Train 2020.

40. Koh IJ, Kang BM, Kim MS, Choi KY, Sohn S, In Y. How Does Preoperative Central Sensitization Affect Quality of Life Following Total Knee Arthroplasty? J Arthrop 2020.

41. Nijs J, Malfliet A, Ickmans K, Baert I, Meeus M. Treatment of central sensitization in patients with 'unexplained'chronic pain: an update. Expert Opin Pharmacother. 2014;15(12):1671-83.

42. Nijs J, Meeus M, Van Oosterwijck J, Roussel N, De Kooning M, Ickmans K, Matic M. Treatment of central sensitization in patients with 'unexplained'chronic pain: what options do we have? Expert opinion on pharmacotherapy. 2011;12(7):1087-98.

43. Ho K-Y, Tay W, Yeo M-C, Liu H, Yeo S-J, Chia S-L, Lo N-N. Duloxetine reduces morphine requirements after knee replacement surgery. $\mathrm{Br} J$ Anaesth. 2010;105(3):371-6.

44. YaDeau JT, Brummett CM, Mayman DJ, Lin Y, Goytizolo EA, Padgett DE, Alexiades MM, Kahn RL, Jules-Elysee KM, Fields KG. Duloxetine and subacute pain after knee Arthroplasty when added to a multimodal analgesic RegimenA randomized, placebo-controlled, triple-blinded trial. J Am Soc Anesthesiol. 2016:125(3):561-72.

45. Chan A-W, Tetzlaff JM, Gøtzsche PC, Altman DG, Mann H, Berlin JA, Dickersin K, Hróbjartsson A, Schulz KF, Parulekar WR. SPIRIT 2013 explanation and elaboration: guidance for protocols of clinical trials. BMJ 2013, 346.

46. Terluin B, Brouwers EP, van Marwijk HW, Verhaak PF, van der Horst HE. Detecting depressive and anxiety disorders in distressed patients in primary care; comparative diagnostic accuracy of the Four-Dimensional Symptom Questionnaire (4DSQ) and the Hospital Anxiety and Depression Scale (HADS). BMC Fam Pract. 2009;10(1):58.

47. Neblett R, Cohen H, Choi Y, Hartzell MM, Williams M, Mayer TG, Gatchel RJ. The Central Sensitization Inventory (CSI): establishing clinically significant values for identifying central sensitivity syndromes in an outpatient chronic pain sample. J Pain. 2013;14(5):438-45.

48. Tanaka K, Nishigami T, Mibu A, Manfuku M, Yono S, Shinohara Y, Tanabe A, Ono R. Validation of the Japanese version of the Central Sensitization Inventory in patients with musculoskeletal disorders. PLoS One. 2017;12(12): e0188719.

49. Bang $H, N i L$, Davis CE. Assessment of blinding in clinical trials. Controlled Clin Trials. 2004;25(2):143-56.

50. Blikman T, Rienstra W, van Raaij $T$, ten Hagen A, Dijkstra B, Zijlstra W, Bulstra $\mathrm{S}$, van den Akker-Scheek I, Stevens M. Duloxetine in OsteoArthritis (DOA) study: study protocol of a pragmatic open-label randomised controlled trial assessing the effect of preoperative pain treatment on postoperative outcome after total hip or knee arthroplasty. BMJ open. 2016:6(3):e010343.

51. Collins SL, Moore RA, McQuay HJ. The visual analogue pain intensity scale: what is moderate pain in millimetres? Pain 1997, 72(1-2):95-97.

52. Farrar JT, Young JP Jr, LaMoreaux L, Werth JL, Poole RM. Clinical importance of changes in chronic pain intensity measured on an 11-point numerical pain rating scale. Pain. 2001;94(2):149-58.

53. Cleeland C, Ryan K. Pain assessment: global use of the Brief Pain Inventory. An Acad Med Singapore 1994

54. De Groot IB, Favejee MM, Reijman M, Verhaar JA, Terwee CB. The Dutch version of the Knee Injury and Osteoarthritis Outcome Score: a validation study. Health Qual Life Outcomes. 2008;6(1):16

55. Giesinger JM, Hamilton DF, Jost B, Behrend H, Giesinger K. WOMAC, EQ-5D and knee society score thresholds for treatment success after total knee arthroplasty. J Arthroplast. 2015;30(12):2154-8.

56. Kahlenberg CA, Nwachukwu BU, McLawhorn AS, Cross MB, Cornell CN, Padgett DE. Patient satisfaction after total knee replacement: a systematic review. HSS Journal ${ }^{\oplus}$. 2018;14(2):192-201.

57. Chow S-C, Shao J, Wang H, Lokhnygina Y: Sample size calculations in clinical research: CRC Press; 2017. 
58. Machin D, Campbell MJ, Tan SB, Tan SH: Sample sizes for clinical, laboratory and epidemiology studies: John Wiley \& Sons; 2018.

59. Al-Sunduqchi MS. Determining the appropriate sample size for inferences based on the Wilcoxon statistics. 1992.

60. Moher D, Hopewell S, Schulz KF, Montori V, Gøtzsche PC, Devereaux P, Elbourne D, Egger M, Altman DG. CONSORT 2010 explanation and elaboration: updated guidelines for reporting parallel group randomised trials. Int J Surg. 2012;10(1):28-55.

\section{Publisher's Note}

Springer Nature remains neutral with regard to jurisdictional claims in published maps and institutional affiliations.

Ready to submit your research? Choose BMC and benefit from:

- fast, convenient online submission

- thorough peer review by experienced researchers in your field

- rapid publication on acceptance

- support for research data, including large and complex data types

- gold Open Access which fosters wider collaboration and increased citations

- maximum visibility for your research: over $100 \mathrm{M}$ website views per year

At $\mathrm{BMC}$, research is always in progress.

Learn more biomedcentral.com/submissions 